\title{
Study of the interaction of DNA and histones by spin-stretching and droplet evaporation
}

\author{
LIU YuYing ${ }^{*}$, WANG PengYe ${ }^{2}$, DOU ShuoXing ${ }^{2}$, ZHANG WeiWei $^{1}$, WANG XueJin $^{1} \&$ \\ SANG Hong $\mathrm{Yi}^{1}$ \\ ${ }^{1}$ College of Science, China Agricultural University, Beijing 100083, China; \\ ${ }^{2}$ Key Laboratory of Soft Matter Physics and Beijing National Laboratory for Condensed Matter Physics, Institute of Physics, Chinese Academy of \\ Sciences, Beijing 100190, China
}

Received September 25, 2010; accepted December 17, 2010

\begin{abstract}
Molecular combing is a powerful and simple method for aligning DNA molecules onto a surface. Using this technique combined with fluorescence microscopy, DNA-histone complexes are stretched on a hydrophobic polymethyl methacrylate (PMMA) surface and observed directly. We have developed a new method to stretch single DNA-histone complexes, termed spin-stretching. The results show that the histones markedly enhance DNA binding to the PMMA surface. DNA winds around the histones and therefore decreases in length. The number of histones that bind to each DNA molecule is found to correlate with the histone concentration. The combed DNA-histone complexes are found to depend on two factors: the binding force on the surface and the centrifugal force at its local position. $\mathrm{Na}^{+}$ions should compete with histones for binding to DNA; however, the observed competitive binding effect of $\mathrm{Na}^{+}$ions at low concentrations was negligible.
\end{abstract}

molecular combing, histone, DNA-histone complex, spin-stretching, droplet evaporation

Citation: $\quad$ Liu Y Y, Wang P Y, Dou S X, et al. Study of the interaction of DNA and histones by spin-stretching and droplet evaporation. Chinese Sci Bull, 2011, 56: 1234-1240, doi: 10.1007/s11434-011-4422-2

DNA, the carrier of genetic information, is the central molecule of life. When free in solution, DNA behaves as a random elastic coil. DNA stretching has recently become an important method to examine the linearity of this macromolecule. Several physical methods have been employed to stretch single DNA molecules, such as magnetic tweezers [1-3], laser tweezers [4-7], atomic force microscopy [8,9], micropipetting [10] and the molecular combing method [11-13].

Molecular combing is a novel approach for stretching DNA molecules. In recent years, many combing methods have been developed for stretching DNA. Such methods include dynamic molecular combing (meniscus moving on a coverslip [14]), spin-stretching [8,9], the evaporation of small droplets of DNA solutions [15,16], the stretching of DNA with a cover slip [17], the mechanical movement of a

\footnotetext{
*Corresponding author (email: 51888lyy@ sina.com)
}

meniscus [18], droplet motion driven by a nitrogen gas flow [19], the precise control of the meniscus motion [20], the ordered stretching of DNA between micro fabricated polystyrene lines [21], the moving droplet method [22] and aligning DNA molecules on a $\mathrm{SiO}_{2}$ surface using a diamond-like carbon (DLC) thin film [23]. Combined with other techniques, the applications of the molecular combing method can be widened to include positioning genes on chromosomes [14], the genomic studies of DNA replication [24], the observation of transcription on a single combed DNA [25], the study of the interaction between DNA and enzymes [26,27] and the self-organized DNA network between two cover slips [28].

Eukaryotic genes do not exist as naked DNA molecules in the nucleus of a cell. Instead, they combine with particular proteins, especially the basic proteins called histones, to form a substance known as chromatin [29]. Chromatin controls gene activity and the inheritance of traits. The fun- 
damental subunit of chromatin is the nucleosome, which consists of approximately 165 base pairs (bp) of DNA wrapped into two superhelical turns around an octamer of core histones (two of each histone H2A, H2B, H3 and H4). This results in a five- to ten-fold compaction of DNA. Chromatin proteins and DNA are partners that control the activities of the genetic material within cells [30].

Protein mediated DNA bending has been studied with various techniques. Atomic force microscopy was used for observing conformations of DNA single molecules or DNA-protein complexes, providing important information about protein locations on DNA and DNA bend angles. The compaction of single DNA molecules by purified histones was studied using magnetic tweezers [31]. Single-molecule measurements using an optical trap have revealed that the wrapping and unwrapping of DNA between 1 and 1.75 turns occurs reversibly. Recently developed fast-scanning AFM has the ability to investigate DNA and nucleosome dynamics in solution. Single-molecule fluorescence resonance energy transfer (FRET) techniques have detected a local dissociation of DNA from the histone core particle that occurs on the sub-second time scale [32]. Using a Brownian dynamics simulation, the interaction of DNA with histone was numerically studied [33]. With chromatin immunoprecipitation (ChIP) and promoter DNA microarray analyses (ChIP-on-chip), the variations of acetylation of histone $\mathrm{H} 3$ in all-trans retinoic acid (RA) induced neuronal cell differentiation have been analyzed [34].

In this work, DNA-histone complexes were stretched on a hydrophobic polymethyl methacrylate (PMMA) surface by two different molecular combing methods (spin-stretching and droplet evaporation), and directly observed with fluorescence microscopy. The two combing methods should be suitable to study the binding properties between DNA and histones at the single-molecule level.

In a previous study, single molecule techniques such as laser trapping and magnetic tweezers have been used to study DNA-histone interactions, and for manipulating DNA molecules tethered to beads. However, such approaches involved extensive manipulations and are inappropriate for high-throughput analysis. In our combing experiment, DNA-histone complexes were stretched by spinning, and large numbers of DNA-histone complexes were combed onto the same surface and analyzed simultaneously. These two combing methods were simple and effective, and most importantly, they could directly study the binding properties between DNA and histones.

For observation with fluorescence microscopy, lambda DNA was labeled with a fluorescent dye YOYO-1 before the reaction and the DNA-histone complexes were then stretched onto the PMMA hydrophobic surface. The interaction between DNA and histones can be analyzed by observing the length and the density of the stretched DNA-histone complexes. In addition, because both DNA and histones are macromolecules, the interaction between them is complex, because many factors influence this interaction. This method will aid investigation into the interaction between DNA and histones at the single molecule level. Currently, characterizing the interaction between DNA and histones from a physics perspective is challenging, and therefore it remains an unsolved problem awaiting a theoretical and experimental approach.

\section{Materials and methods}

\subsection{Experimental materials and equipment}

Lambda DNA was purchased from Sino-American Biotechnology (Beijing, China). Fluorescent dye and the oxazole yellow dimer (YOYO-1) were purchased from Molecular Probes (USA). Bis-Tris and histones were purchased from Sigma (USA). All buffers were made with ultra-pure water purified through a Milli-Q water purification system (Millipore Corporation, France). Fluorescently stained DNA molecules were observed using an inverted fluorescence microscope (IX-70; Olympus). The images were captured by a cooled CCD camera (CoolSNAP-HQ, Roper Scientific Inc). MetaMorph software (Universal Imaging Corporation) was used for the system control, data acquisition and data processing.

\subsection{Surface treatment}

Quartz plates (21 mm× $42 \mathrm{~mm}$ ) were immersed in $\mathrm{NaOH}$ $(0.5 \mathrm{~mol} / \mathrm{L})$ for $\sim 10 \mathrm{~min}$, and then rinsed thoroughly in ultra-pure water (Millipore S.A., France). Quartz surfaces were rendered hydrophobic by coating the surfaces with PMMA. A droplet (0.2-0.3 mL) of PMMA (560F, Japan) in chloroform $(10 \%(\mathrm{wt} / \mathrm{wt}))$ was dripped down onto the center of a cleaned quartz surface, which was mounted horizontally on the spin-coating machine using double-sided tape, and spread by spin-coating at $5500 \mathrm{r} / \mathrm{min}$ for $1 \mathrm{~min}$. After spincoating, the PMMA film was formed evenly on the whole surface. The quartz plate was then baked at $145^{\circ} \mathrm{C}$ for $\sim 30$ min and stored at room temperature in a dust-free environment. During the preparation of the PMMA film, it was very important to maintain the uniformity of the PMMA solution and the cleanliness of the surface.

\subsection{DNA and DNA-histone complex preparation}

Lambda DNA (48.5 kb) was stained with a fluorescent dye, oxazole yellow dimer (YOYO-1, Molecular Probes) at a ratio of ten base pairs per one dye molecule $(\mathrm{bp} /$ dye $=10)$ by mixing the DNA sample with a specific volume of freshly prepared $0.1 \mu \mathrm{mol} / \mathrm{L}$ dye solution $\left(10 \mathrm{mmol} \mathrm{L}^{-1}\right.$ Tris/1 mmol L ${ }^{-1}$ EDTA buffer, pH 8.0). The DNA/YOYO-1 solution was incubated for approximately $30 \mathrm{~min}$ in the dark, and then diluted to $6.5 \mathrm{pmol} / \mathrm{L}$ in bis-Tris buffer $(50$ $\mathrm{mmol} / \mathrm{L}, \mathrm{pH} \sim 5.6)$. 
Histones were diluted using a bis-Tris buffer $(50 \mathrm{mmol} / \mathrm{L}$, $\mathrm{pH} \sim 6.6)$. DNA ( $6.5 \mathrm{pmol} / \mathrm{L})$ and diluted histones were incubated together at $37^{\circ} \mathrm{C}$ for $30 \mathrm{~min}$. The concentration ratio of DNA to histone ranged between 10 to 50 .

\subsection{Spin-stretching DNA and the DNA-histone complex}

The quartz glass surface coated with PMMA was mounted horizontally on the spin-coating machine using double-sided tape. A series of DNA droplets $(5-8 \mu \mathrm{L})$ was deposited onto the hydrophobic PMMA surface 5 min before spin-coating. This procedure makes one end of the DNA molecule suitable for binding to the substrate. The distances between the droplets and rotating center were 1 and $2 \mathrm{~cm}$. The PMMA surface was then accelerated to its final rotational speed of $2000-6000 \mathrm{r} / \mathrm{min}$.

\subsection{Stretching DNA and the DNA-histone complex by droplet evaporation}

Droplets of the sample solution containing DNA $(1.2 \mu \mathrm{L})$ or DNA-histone complexes $(1.2 \mu \mathrm{L})$ were deposited onto an open PMMA surface. After a few minutes, with the droplets drying, a significant number of fixed DNA molecules were fully elongated, aligned radially and concentrated near the peripheries of the spots to give a "sunburst" pattern.

\subsection{DNA Imaging by fluorescence microscopy}

YOYO-1 has an excitation maximum at $491 \mathrm{~nm}$ and emission maximum at $509 \mathrm{~nm}$; that is, YOYO-1 molecules emit green fluorescence under the excitation of blue light. DNA molecules can be seen by observing the fluorescence of YOYO-1. A $100 \mathrm{~W}$ mercury lamp was used in combination with a U-MWB excitation cube (BP450-480, DM500, BA515). Fluorescently stained DNA molecules were observed using an inverted optical microscope (IX-70; Olympus) by epifluorescence and a $20 \times$ objective. The CCD acquisition time used was three seconds.

\section{Results}

\subsection{Stretching DNA and DNA-histone complexes with droplet evaporation}

The concentration of histones was 50-fold higher then DNA. DNA and histones were incubated together at $37^{\circ} \mathrm{C}$ for $1 \mathrm{~h}$. DNA-histone complexes were combed by the droplet evaporation method. The results were as follows: the length of the combed DNA-histone complexes became shorter, which indicated that the histones had combined with the DNA molecules, and the distribution of combed DNA-histone complexes was denser. As the concentration of histones increased, more histones were observed to bind to each
DNA molecule. The histones enhanced the DNA binding affinity to the hydrophobic surface (Figure 1).

Using the droplets evaporation combing method, the interaction of histones with the DNA led to a noticeable reduction in the length of the DNA. Moreover, the DNA condensed at high histone concentrations.

From Figure 1, we measured the mean length of DNA as $\sim 22 \mu \mathrm{m}$, whereas the mean length of the DNA-histone complex was $\sim 13 \mu \mathrm{m}$. DNA winds around histone complexes 1.75 turns and involves $146 \mathrm{bp}$, which corresponds to a DNA length of $0.05 \mu \mathrm{m}$. Consequently, we concluded that when the concentration ratio of histone to DNA was 50, the average number of histones binding to a single DNA molecule was about 188 .

Lambda DNA (48.5 kb) was stained with YOYO-1 in our experiments. To reduce the illumination time and thus the fluorescence photobleaching of YOYO-1, we generally started our fluorescence microscopy observations after the droplets had dried completely. In addition, this also avoided photocleavage of the DNA in the solution. As the DNA/YOYO-1 solution was incubated for only about 30 min, the dominant binding mode of YOYO-1 should involve external binding via electrostatic interactions. As such, the positively charged YOYO-1 dye should reduce the binding affinity of the positively charged histones by competitively binding to the DNA. We found that YOYO- 1 affected DNA-histone interactions to some degree. As the amount of YOYO-1 bound to the DNA increased, fewer histone molecules were bound to the DNA. So in all of our experiments, we selected a ratio of ten base pairs per dye molecule $(\mathrm{bp} /$ dye $=10)$. We used the following reaction conditions: the concentration ratio of histone to DNA was

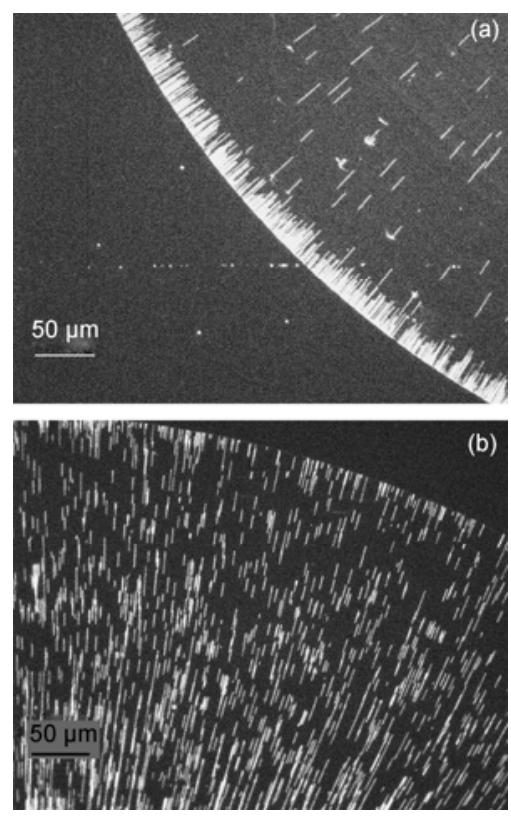

Figure 1 (a) Image of combed DNA molecules (6.5 pmol/L); (b) image of combed DNA-histone complexes. The concentration of histones was 50-fold higher than the DNA. 
between 5 and 100 , the temperature was $37^{\circ} \mathrm{C}$, the ratio of base pairs to dye molecules was $10(\mathrm{bp} / \mathrm{dye}=10)$ and the $\mathrm{pH}$ value of the buffer was lower than 7 [35].

\subsection{Spin-stretching of the DNA-histone complex}

The interaction of DNA and histones has been studied by the droplets evaporation combing method. With droplet evaporation, the DNA-histone complexes were stretched by the force of the fluid flow as the droplet evaporated; thus in practice, the magnitude of this force cannot be modulated or controlled.

At different histone and DNA concentration ratios, the conformation of the DNA-histone complex may vary. Force-induced nucleosome disruption was clearly observed by the optical trap method. In our experiment, it is possible that during the stretching process the interaction between the histones and DNA is disrupted.

To further investigate the interaction between the DNA and histones, we stretched lambda DNA and the DNAhistone complexes on the PMMA surface using a new method, spin-stretching, and examined the density and length of the combed molecules by fluorescence microscopy. The experimental conditions are as follows: the concentration of DNA was $6.5 \mathrm{pmol} / \mathrm{L}$, the $\mathrm{pH}$ was 5.6 , the rotational speed was $2000 \mathrm{r} / \mathrm{min}$ and the distance between the droplet and the rotating center was $1 \mathrm{~cm}$. The concentration ratios of histone to DNA were 10, 30 and 50. The results showed that the number of combed DNA molecules (without histones) was not significant (Figure 2(a)). Here the DNA binding force to the surface was not strong, and many DNA molecules were dislodged from the surface when spinning because of the centrifugal force. The lengths of the combed DNA-histone complexes were significantly shorter than that of combed DNA when the concentration ratio was 10 (Figure 2(b)).

However, under the same conditions with the number of histones increasing, we found a series of very interesting phenomena. When the concentration ratio of histone to DNA was 30, the combed DNA-histone complexes became shorter and more densely distributed (Figure 3(a)). When the ratio of histone to DNA was 50, the DNA-histone complexes were not stretched, and they primarily adopted random coiled shapes. The numbers of combed DNA-histone complexes increased significantly (Figure 3(b)).

These phenomena showed that histones enhanced DNA binding capacity to the surface. From the above results, we suggest that as more histones bind to the DNA, this induces DNA to wrap more tightly around the histones, and the force between DNA and histones increases, thereby leading to a complex which was difficult to unwind and stretch. The mean lengths of DNA and DNA-histone complexes were measured (Figure 4).

For two of the ratio groups (concentration ratio was 30 and 50), we continued to increase the rotation speed. When
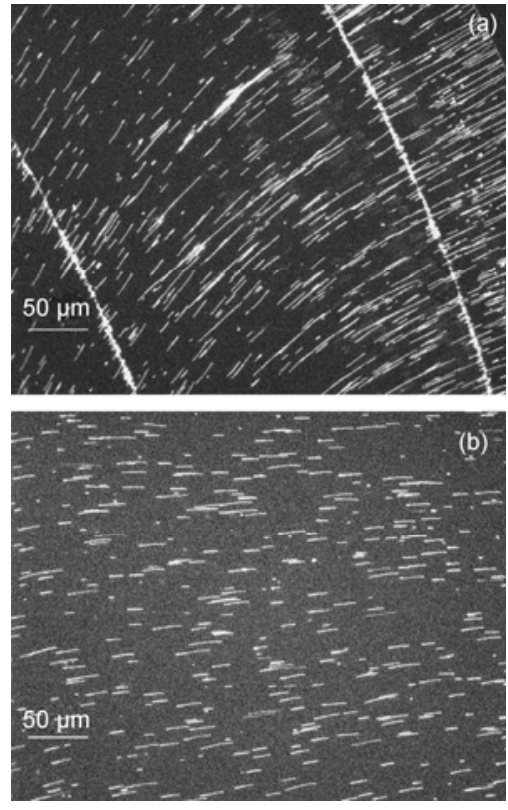

Figure 2 (a) Image of the combed DNA (6.5 pmol/L); (b) image of the combed DNA-histone complexes. The concentration of histone was 10fold higher than DNA.
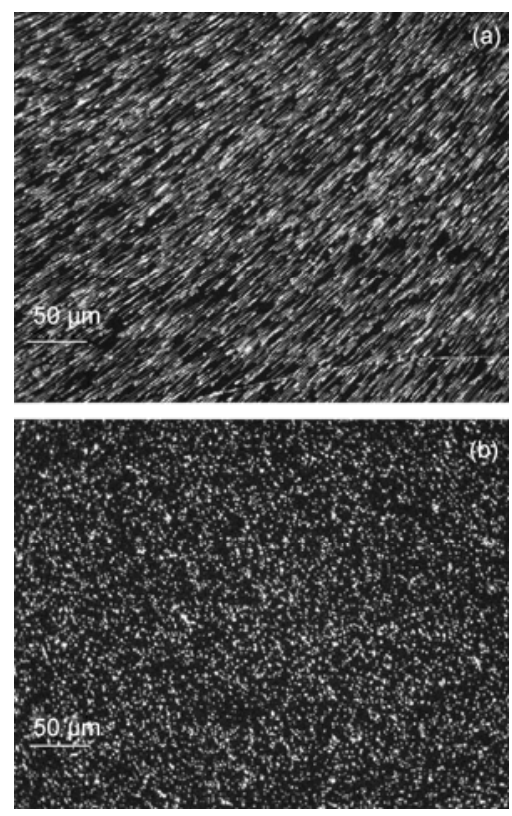

Figure 3 Image of the combed DNA-histone complexes. (a) The concentration of histones was 30-fold higher than the DNA; (b) the concentration of histones was 50-fold higher than the DNA. Rotating speed: $2000 \mathrm{r} / \mathrm{min}$.

the rotating speed was $6000 \mathrm{r} / \mathrm{min}$, the DNA-histone complexes (concentration ratio was 30) were stretched and elongated and adopted a linear shape (Figure 5(a)). At the same time, the number of combed DNA-histone complexes became fewer than that shown in Figure 3(a) because of the higher centrifugal force. However, when the concentration ratio was 50:1, only a few DNA-histone complexes were stretched and adopted a linear shape; most molecules 


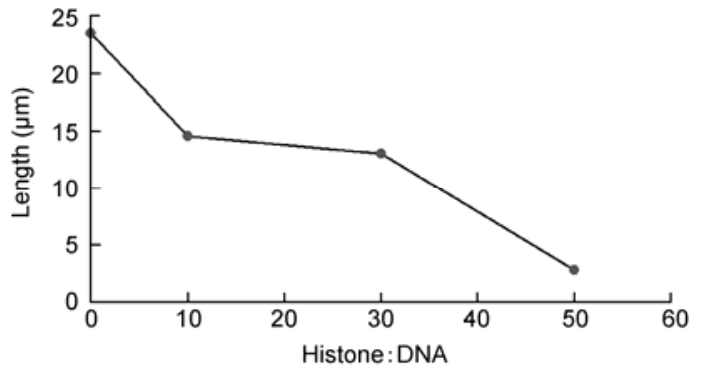

Figure 4 The mean length of DNA and DNA-histone complexes at different DNA: histone ratios.
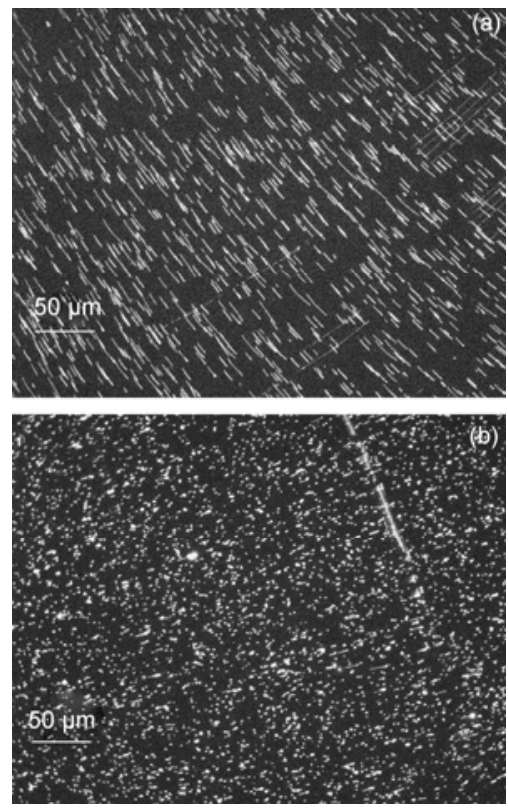

Figure 5 Image of the combed DNA-histone complexes. (a) The concentration of histones was 30-fold higher than the DNA concentration; (b) the concentration of histones was 50-fold higher than the DNA concentration. Rotating speed: $6000 \mathrm{r} / \mathrm{min}$.

showed coiled shapes (Figure 5(b)). We conclude that as the histone concentration increased more histones were bound to the DNA molecules. At the same rotating speed, as the number of histones bound to the DNA increased, the DNA appears to wrap around the histones more tightly. In addition, these histone-rich-DNA complexes were found to adhere to the surface more tightly and the DNA-histone complexes condensed and could not be stretched fully.

\subsection{Spin-stretching of the DNA-histone- $\mathrm{Na}^{+}$complex}

For the DNA-histone complexes with a concentration ratio $50, \mathrm{NaCl}$ was added and the complexes stretched by spinning. DNA (6.5 pmol/L), $\mathrm{NaCl}(20 \mathrm{mmol} / \mathrm{L})$ and histones $\left(3.25 \times 10^{-10} \mathrm{~mol} / \mathrm{L}\right)$ were incubated together for $1 \mathrm{~h}$ at $37^{\circ} \mathrm{C}$. At lower rotating speeds (2000 and $4000 \mathrm{r} / \mathrm{min}$ ), the DNAhistone- $\mathrm{Na}^{+}$complexes were not stretched and showed predominantly coil shapes. There is almost no difference between the combed DNA-histone- $\mathrm{Na}^{+}$complexes and the DNA-histone complexes at lower rotating speeds. However, when the rotating speed was $6000 \mathrm{r} / \mathrm{min}$, there were more stretched DNA-histone- $\mathrm{Na}^{+}$complexes compared to the number of stretched DNA-histone complexes (Figures 6 and 5 (b)). In our experiment, the concentration of $\mathrm{NaCl}(20$ $\mathrm{mmol} / \mathrm{L}$ ) was low. However, $\mathrm{Na}^{+}$occupied some binding sites on DNA for histones, thereby reducing the number of histones interacting with the DNA. Genomic DNA is well known to be densely packed inside the cell nucleus and viral capsids. Such close packing suggests that electrostatic repulsion between negatively charged DNA in the condensed state is balanced by counterion-induced attractions. The effective attraction between DNA in trivalent and quadrivalent electrolytes is documented. DNA attraction has been observed for divalent cations: bridged by $\mathrm{Mg}^{2+}$ ions, DNA interlocks in the minor-grove-to-minor-grove conformation. Monovalent ions only modulate the repulsive interactions between DNA. The effect of $\mathrm{Na}^{+}$on the DNA-histone complex is considered to be weak, while the concentration of added $\mathrm{Na}^{+}$is very low $(20 \mathrm{mmol} / \mathrm{L})$. With increasing $\mathrm{NaCl}$ concentrations, the competitive role of $\mathrm{Na}^{+}$to inhibit the interaction of histones with DNA would be stronger.

We also found that under the same conditions, except for the rotating radius (i.e. a larger radius), longer DNA-histone$\mathrm{Na}^{+}$complexes were observed (Figure 6(b)). This indicates that the stretching of the DNA complexes was related to its local linear velocity.

We also measured the mean length of the DNA-histone complex and the DNA-histone- $\mathrm{Na}^{+}$complex at identical
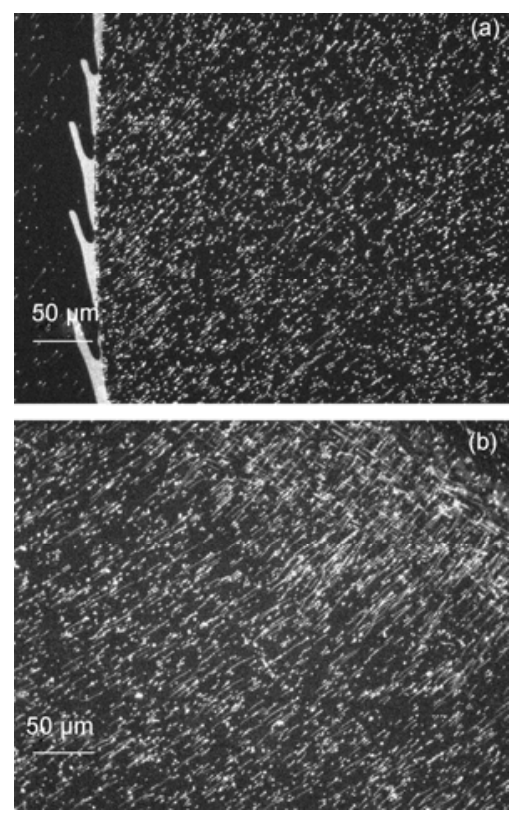

Figure 6 Image of the combed DNA-histone- $\mathrm{Na}^{+}$complexes. The concentration of histones was 50-fold higher than DNA. Rotating speed: 6000 $\mathrm{r} / \mathrm{min}$. (a) Distance from the rotating center: $r=1 \mathrm{~cm}$; (b) distance from rotating center: $r=2 \mathrm{~cm}$. 


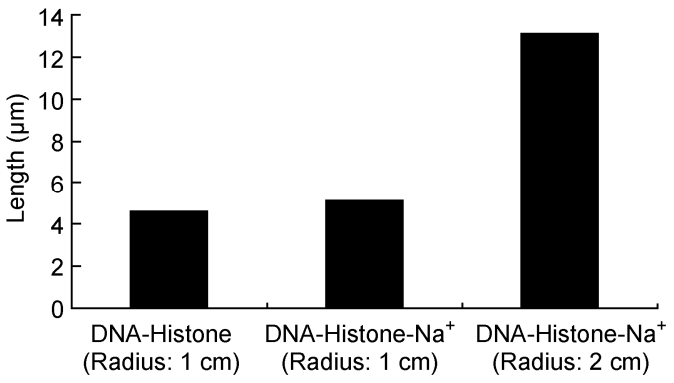

Figure 7 The mean length of the DNA-histone complex and the DNAhistone- $\mathrm{Na}^{+}$complex at the same rotating speed $(6000 \mathrm{r} / \mathrm{min})$.

rotating speeds $(6000 \mathrm{r} / \mathrm{min})$; however, the radii differed. The concentration of $\mathrm{Na}^{+}$was $20 \mathrm{mmol} / \mathrm{L}$. The results showed that the combed DNA-histone and DNA-histone$\mathrm{Na}^{+}$(radius $1 \mathrm{~cm}$ ) have no obvious differences. However, the mean length of the combed DNA-histone- $\mathrm{Na}^{+}$complex became much larger at a radius of $2 \mathrm{~cm}$ (Figure 7). $\mathrm{Na}^{+}$ competes with histones for DNA binding, yet such an effect appears to be negligible.

\section{Conclusions}

We studied the interaction between DNA and histones using a spin-stretching combing method and droplet evaporation. Some conclusions are drawn.

(1) Adding different concentrations of histones into the DNA solution modulated the number of histones bound to the DNA, thus changing the degree of DNA wrapped around histones. With increasing amounts of histones bound to the DNA, the force between the DNA and histones became much stronger. In addition, the DNA-histone complex was difficult to stretch. At the same time, the interaction with the histones enhanced the force between the DNA and the hydrophobic surface.

(2) The combed DNA-histone complexes depend on two kinds of force: the binding force on the surface and the centrifugal force at its local position. The centrifugal force depends on the rotating speed, rotating radius, or local linear velocity.

(3) When $\mathrm{Na}^{+}$ions were added into the DNA-histone solution, the presence of the $\mathrm{Na}^{+}$ions did not significantly affect the interaction between the histones and DNA.

This work was supported by the National Natural Science Foundation of China (60025516 and 10334100) and the Scientific Research Start-up Foundation of China Agricultural University (2009-2-05).

1 Smith S B, Finzi L, Bustamante C. Direct mechanical measurements of the elasticity of single DNA molecules by using magnetic beads. Science, 1992, 258: 1122-1126

2 Strick T R, Allemand J F, Bensimon D, et al. The elasticity of a single supercoiled DNA molecule. Science, 1996, 271: 1835-1837

3 Allemand J F, Bensimon D, Lavery R, et al. Stretched and over- wound DNA forms a pauling-like structure with exposed bases. Proc Natl Acad Sci USA, 1998, 95: 14152-14157

4 Perkins T T, Smith D E, Chu S. Direct observation of tube-like motion of a single polymer chain. Science, 1994, 264: 819-822

5 Perkins T T, Quake S R, Smith D E, et al. Relaxation of a single DNA molecule observed by optical microscopy. Science, 1994, 264 : $822-826$

6 Smith S B, Cui Y J, Bustamante C. Overstretching B-DNA: The elastic response of individual double-stranded and single-stranded DNA molecules. Science, 1996, 271: 795-799

7 Bennink M L, Schärer O D, Kanaar R, et al. Single-molecule manipulation of double-stranded DNA using optical tweezers: Interaction studies of DNA with RecA and YOYO-1. Cytometry, 1999, 36: 200208

8 Ye J Y, Umemura K, Ishikawa M, et al. Atomic force microscopy of DNA molecules stretched by spin-coating technique. Anal Biochem, 2000, 281: 21-25

9 Yokota H, Sunwoo J, Sarikaya M, et al. Spin-stretching of DNA and protein molecules for detection by fluorescence and atomic force microscopy. Anal Chem, 1999, 71: 4418-4422

10 Cluzel P, Lebrun A, Heller C, et al. DNA: An extensible molecule. Science, 1996, 271: 792-794

11 Bensimon A, Simon A, Chiffaudel A, et al. Alignment and sensitive detection of DNA by a moving interface. Science, 1994, 265: 20962098

12 Liu Y Y, Wang P Y, Dou S X, et al. Study the effects of metallic ions on the combination of DNA and histones with molecular combing technique. Chinese Sci Bull, 2005, 50: 731-737

13 Zhang Y, Chen S F, Ouyang Z Q, et al. Stretching and imaging studies of single DNA molecules. Chinese Sci Bull, 2000, 45: 1365-1368

14 Michalet X, Ekong R, Fougerousse F, et al. Dynamic molecular combing: stretching the whole human genome for high-resolution studies. Science, 1997, 277: 1518-1523

15 Wang W N, Lin J Y, Schwartz D C. Scanning force microscopy of DNA molecules elongated by convective fluid flow in an evaporating droplet. Biophys J, 1998, 75: 513-520

16 Jing J P, Huang J, Hu X H, et al. Automated high resolution optical mapping using arrayed, fluid-fixed DNA molecules. Proc Natl Acad Sci USA, 1998, 95: 8046-8051

17 Kraus J, Weber R G, Cremer M, et al. High-resolution comparative hybridization to combed DNA fibers. Am J Human Genet, 1997, 99: 374-380

18 Yokota H, Johnson F, Lu H B, et al. A new method for straightening DNA molecules for optical restriction maping. Nucl Acids Res, 1997, 25: $1064-1070$

19 Li J W, Bai C L, Wang C, et al. A convenient method of aligning large DNA molecules on bare mica surfaces for atomic force microscopy. Nucl Acids Res, 1998, 26: 4785-4786

20 Otobe K, Ohtani T. Behavior of DNA fibers stretched by precise meniscus motion control. Nucl Acids Res, 2001, 29: e109

21 Klein D C G, Gurevich L, Janssen J W, et al. Ordered stretching of single molecules of deoxyribose nucleic acid between microfabricated polystyrene lines. Appl Phys Lett, 2001, 78: 2396-2398

22 Oshige M, Yamaguchi K, Matsuura S, et al. A new DNA combing method for biochemical analysis. Anal Biochem, 2010, 400: 145-147

23 Kim H J, Yun D Y, Choi W S, et al. Selective assembly of DNA using DLC film as passivation layer for the application to nano-device. Diam Relat Mater, 2009, 18: 1015-1018

24 Herrick J, Jun S, Bechhoefer J, et al. Kinetic model of DNA replication in Eukaryotic organisms. J Mol Biol, 2002, 320: 741-750

25 Gueroui Z, Place C, Freyssingeas E. Observation by fluorescence microscopy of transcription on single combed DNA. Proc Natl Acad Sci USA, 2002, 99: 6005-6010

26 Taylor J R, Fang M M, Nie S M. Probing specific sequences on single DNA molecules with bioconjugated fluorescent nanoparticles. Anal Chem, 2000, 72: 1979-1986

27 Liu Y Y, Wang P Y, Dou S X, et al. Direct visualization of RecQ helicase-DNA interaction with fluorescence microscopy and atomic force microscopy. Sci Tech Adv Mater, 2005, 6: 842-847 
28 TakedaY, Mafune F, Kondow T. Self-organization of histone-jointed three-dimensional DNA network. Mater Sci Eng C, 2004, 24: 769-773

29 Weaver R F. Molecular Biology. 2nd ed. Beijing: Science Press, 2002. 380-387

30 Felsenfeld G, Groudine M. Controlling the double helix. Nature, 2003, 421: 448-453

31 Ran S Y, Wang X L, Fu W B, et al. Single molecule DNA compaction by purified histones. Chinese Sci Bull, 2008, 53: 836-841

32 Suzuki Y K, Higuchi Y J, Hizume K, et al. Molecular dynamics of DNA and nucleosomes in solution studied by fast-scanning atomic force microscopy. Ultromiscropy, 2010, 110: 682-688

33 Li W, Dou S X, Wang P Y. Brownian dynamics simulation of nucleosome formation and disruption under stretching. J Theor Biol, 2004, 230: $375-383$

34 Fang H B, Mi Y, Wu N H, et al. Genome-wide distribution of histone $\mathrm{H} 3$ acetylation in all-trans retinoic acid induced neuronal differentiation of SH-SY5Y cells. Chinese Sci Bull, 2009, 54: 1043-1049

35 Liu Y Y, Dou S X, Wang P Y, et al. Study the interaction of DNA and histone by molecular combing. Acta Phys Sin, 2005, 54: 622627

Open Access This article is distributed under the terms of the Creative Commons Attribution License which permits any use, distribution, and reproduction in any medium, provided the original author(s) and source are credited. 